\title{
System Identification Applied to Dynamic CFD Simulation and Wind Tunnel Data
}

\author{
Patrick C. Murphy ${ }^{1}$ \\ NASA Langley Research Center, Hampton, VA, 23681-2199 \\ Vladislav Klein ${ }^{2}$ \\ National Institute of Aerospace, NASA Langley Research Center, Hampton, VA, 23681-2199 \\ Neal T. Frink ${ }^{3}$ \\ NASA Langley Research Center, Hampton, VA, 23681-2199 \\ Dan D. Vicroy ${ }^{4}$ \\ NASA Langley Research Center, Hampton, VA, 23681-2199
}

\begin{abstract}
Demanding aerodynamic modeling requirements for military and civilian aircraft have provided impetus for researchers to improve computational and experimental techniques. Model validation is a key component for these research endeavors so this study is an initial effort to extend conventional time history comparisons by comparing model parameter estimates and their standard errors using system identification methods. An aerodynamic model of an aircraft performing one-degree-of-freedom roll oscillatory motion about its body axes is developed. The model includes linear aerodynamics and deficiency function parameters characterizing an unsteady effect. For estimation of unknown parameters two techniques, harmonic analysis and two-step linear regression, were applied to roll-oscillatory wind tunnel data and to computational fluid dynamics (CFD) simulated data. The model used for this study is a highly swept wing unmanned aerial combat vehicle. Differences in response prediction, parameters estimates, and standard errors are compared and discussed.
\end{abstract}

\section{Nomenclature}

\begin{tabular}{|c|c|c|c|c|}
\hline$A_{j}, B_{j}$ & $=$ Fourier coefficients & $\mathrm{T}$ & $=$ & dimensional time constant, sec \\
\hline$a, \mathrm{~b}_{1}, \mathrm{c}$ & deficiency function parameters & $t$ & $=$ & time, sec \\
\hline$b$ & wing span, $\mathrm{ft}$ & $V$ & $=$ & velocity, fps \\
\hline$C_{l}, C_{n}$ & rolling and yawing-moment coefficients & $\alpha$ & $=$ & angle of attack, rad or deg \\
\hline $\mathrm{C}_{\mathrm{Y}}$ & side-force coefficient & $\alpha_{0}$ & $=$ & mean angle of attack, rad or deg \\
\hline $\bar{c}$ & mean aerodynamic chord, $\mathrm{ft}$ & $\beta$ & $=$ & sideslip angle, rad or deg \\
\hline$f$ & frequency, $\mathrm{Hz}$ & $\phi$ & $=$ & roll angle, rad or deg \\
\hline$F_{a \beta}$ & deficiency functions & $\eta$ & $=$ & state variable \\
\hline$k$ & $=$ reduced frequency, $\pi b f / V$ & $\sigma$ & $=$ & standard error \\
\hline$m$ & No. of harmonics in Fourier expansion & $\tau$ & $=$ & dummy integration variable \\
\hline$N$ & number of data points & & & \\
\hline$p, r$ & roll and yaw rates, $\mathrm{rad} / \mathrm{sec}$ & $\tau_{1}$ & $=$ & getso \\
\hline $\begin{array}{l}R^{2} \\
\mathrm{~s}\end{array}$ & $\begin{array}{l}=\text { multiple correlation coefficient } \\
=\text { estimated standard error }\end{array}$ & $\omega$ & $=$ & angular frequency, $\mathrm{rad} / \mathrm{sec}$ \\
\hline & $=$ reference area, $\mathrm{ft}^{2}$ & $\psi$ & $=$ & yaw angle, rad or deg \\
\hline
\end{tabular}

\footnotetext{
${ }^{1}$ Senior Research Engineer, Dynamic Systems \& Control Branch, MS 308, Associate Fellow.

${ }^{2}$ Professor Emeritus, Dynamic Systems \& Control Branch, MS 308, Associate Fellow.

${ }^{3}$ Senior Research Engineer, Configuration Aerodynamics Branch, MS 499, Associate Fellow.

${ }^{4}$ Senior Research Engineer, Flight Dynamics Branch, MS 308, Associate Fellow.
} 


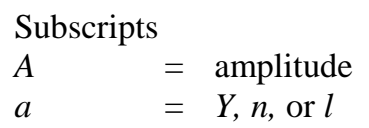

Aerodynamic Derivatives

$$
\begin{aligned}
C_{a_{\beta}} & =\frac{\partial C_{a}}{\partial \beta} \text { where } a=Y, n \text {, or } l \\
C_{a_{p}} & =\frac{b}{2 V} \frac{\partial C_{a}}{\partial p}, C_{a_{r}}=\frac{b}{2 V} \frac{\partial C_{a}}{\partial r}
\end{aligned}
$$

\section{Introduction}

$\mathrm{S}_{\mathrm{n}}^{\mathrm{N}}$ INCE the early days of flight, aerodynamicists have focused on the problem of finding an adequate aerodynamic model for aircraft with good prediction capability. The problem has become more acute over time due to increasing demands from both military and civilian sectors. Demanding mission requirements from the military and safety requirements for civilian aircraft have created a need for more general aerodynamic models that can adequately predict aircraft response even in nonlinear unsteady flight regimes. Researchers have followed two paths to address this modeling problem. A numerical path using Computational Fluid Dynamics (CFD) technology and an experimental path using direct measurements of aircraft responses in flight or scale models in wind tunnels along with System Identification (SID) technology to extract mathematical models. Advances in both computational and experimental technologies have created opportunities for the combination of these technologies to make significant improvements in managing more difficult aircraft modeling problems and reducing costs.

In order to realize this opportunity, one area of modeling research that needs to be addressed more carefully is model validation. The best model validation results are demonstrated by adequate prediction of aircraft responses in flight test. For legitimate validation tests, the validation data should be different from the data used for model identification. When flight test validation data are not available or practical to obtain then CFD predictions and ground-based experimental data are often compared to corroborate the results. Typically this comparison is limited to time history comparisons. A more general comparison can be made by comparing model parameters identified from the time history data. Model parameters are key elements of flight dynamics simulations, stability analysis, and control design. This comparison requires mean values along with uncertainty bounds in order to be statistically accurate. Although it is not common practice for wind tunnel experimentalists or CFD researchers to present results with associated error bounds, some efforts ${ }^{1-3}$ are being made to improve this shortcoming. Although CFD results do not contain random errors, there are potentially large uncertainties due to systematic and modeling errors. Significant uncertainty, for example, can be generated where sensitivities to turbulence models, grid selection, or time steps, are large. Sources of uncertainty for ground-based experiments are well known and can include a variety of systematic and random errors such as direct measurement uncertainty, similitude errors, and modeling errors.

A common denominator that facilitates comparisons of wind tunnel and CFD results is to consider aircraft stability and control $(\mathrm{S} \& \mathrm{C})$ characteristics in the form of a general aerodynamic model identified from those results. For preliminary evaluations, the commonly used in-phase and out-of-phase coefficients that capture important dynamic S\&C frequency characteristics are useful. These preliminary evaluations of S\&C characteristics can be made from well established conventional dynamic wind tunnel testing methods. This testing provides time histories of aerodynamic coefficients of an aircraft scale model while undergoing one-degree-of freedom forced oscillations about its body axes. Test conditions typically include selected angle of attack, amplitude, and frequency conditions. For dynamic testing, Reynolds number selection is typically limited to capabilities of the wind tunnel and dynamic test rig. Analysis of these data provides conventional in-phase and out-of-phase coefficients.

Because of recent participation by NASA ${ }^{3,4}$ in a 3-year NATO/RTO AVT-161 Task Group titled "Assessment of Stability and Control Prediction Methods for NATO Air \& Sea Vehicles", a unique opportunity is afforded to the authors to explore and validate a range of strategies for creating CFD-derived nonlinear flight simulation models within a collaborative international environment. The focus of AVT-161 was to investigate the applicability of current CFD tools for predicting S\&C characteristics of air and sea vehicles. As summarized in Ref. 5, the team made remarkable progress through the leveraged efforts of many engineers and researchers from 9 countries by 1) conceiving a generic unmanned combat air vehicle (UCAV) focus configuration (Fig. 1) called the Stability and Control Configuration (SACCON), 2) designing and building a wind-tunnel model, 3) conducting two test entries in Germany and one in the United States ${ }^{3,6-8}$, and 4) conducting and coordinating several multinational CFD studies. Experimentalists within the AVT-161 team conducted dynamic wind-tunnel tests on SACCON undergoing forced oscillation in pitch, roll, and yaw to measure the dynamic stability and aerodynamic characteristics ${ }^{3,8}$. Other team members ran parallel dynamic computational studies following a similar approach of modeling SACCON undergoing pitch, roll, and yaw oscillation with their respective CFD tools ${ }^{4,7-14}$. The collective experiences from 
these studies have confirmed the difficulty of achieving accurate and efficient computation of dynamic stability characteristics of highly nonlinear flows, which are highly sensitive to choice of flow solver, characteristic time steps, grid size, and turbulence models. Fortunately, a 3-year follow-on activity to AVT-161 is planned to further explore the CFD modeling issues of the SACCON, and develop the techniques for extracting dynamic stability derivatives for flight simulation models from CFD solutions. Only a limited number of AVT-161 studies addressed the reduced-order modeling of SACCON dynamic S\&C characteristics ${ }^{11,14,15}$.

Since the authors plan to contribute to the AVT-161 follow-on effort, this paper is reporting on a preliminary exploratory study to investigate the aerodynamic modeling of SACCON from wind tunnel data and NASA CFD predictions of aerodynamic responses to linear and nonlinear flows over a range of conditions. This study is focused on model validation using CFD and wind tunnel data, taking into account uncertainty bounds. A second paper is planned for the AVT-189 Specialist Meeting in October 2011 to address model identification methodology.

In the current study, both conventional and advanced modeling methods ${ }^{16-18}$ are applied as appropriate to identify aerodynamic model equations as well as in-phase and out-of-phase coefficients. More general models will capture both steady and unsteady dynamics as well as linear and nonlinear responses. Measurements for identification of the mathematical models will be taken from the two sources. Uncertainty between wind tunnel and CFD will be limited to standard errors obtained from estimation of model parameters using system identification methods. For CFD simulated data, the model uncertainty will reflect a range associated with uncertainty due to modeling error and turbulence model selection; uncertainty related to grid size and time steps choices are not included in this paper.

This paper describes the model identification problem and provides a more general aerodynamic model structure as well as appropriate estimation techniques for analysis of dynamic data. Since standard errors are a natural byproduct of the identification methodology the analysis will facilitate more meaningful comparisons between the two sources of data. This approach suggests the importance of experiment design to allow identification of aerodynamic phenomenon and validation modeling results. Recommendations for future tests that facilitate wind tunnel and CFD comparisons will be provided in the concluding remarks.

\section{Wind Tunnel Measured Data}

A portion of the lateral dynamic data collected for the SACCON model in the NASA Langley $14 \times 22$ Subsonic Tunnel in Hampton, Virginia, was selected for analysis in this study. An internal six-component stain gauge balance was used for the force and moment measurements. The sample rate was $300 \mathrm{~Hz}$ with an anti-alias filter at $100 \mathrm{~Hz}$. Additional digital filtering was performed with a low pass filter at $4 \mathrm{~Hz}$ for this study. The filter was run in both directions to ensure no phase error was added to the data. Frequency effects were explored in roll forced-oscillation for the configuration with a round leading edge and fixed transition (RLE-FT) at tunnel velocity of 60 fps. The selected roll-oscillatory data were obtained at four nominal oscillation angles of attack, $0^{\circ}, 14^{\circ}, 15^{\circ}$, and $20^{\circ}$, at ten different frequencies from $0.24 \mathrm{~Hz}$ to $1.0 \mathrm{~Hz}$, and with amplitude of $5^{\circ}$ in bank angle.

\section{CFD Simulated Data}

CFD simulations were computed to correspond with the same conditions as the forced-oscillation SACCON wind tunnel test. The specific test conditions chosen presented unsteady behavior during wind tunnel roll forcedoscillation tests. Consequently, the case studied will highlight more general modeling and validation comparisons. The CFD methodology is described next, followed by an overview of the solution process.

Computations are performed with the USM3D flow solver ${ }^{19}$ that is part of the NASA Tetrahedral Unstructured Software System (TetrUSS) ${ }^{20}$. USM3D is a parallelized tetrahedral cell-centered, finite volume compressible RANS flow solver. The term "cell centered" means that the finite volume flow solution is solved at the centroid of each tetrahedral cell. Inviscid flux quantities are computed across each tetrahedral cell face using various upwind schemes. Spatial discretization is accomplished by a novel reconstruction process, based on an analytical formulation for computing solution gradients within tetrahedral cells. The solution can be advanced in time by a $2^{\text {nd }}-$ order "physical" time step scheme, a $2^{\text {nd }}$-order "dual" time step scheme, or to a steady-state condition by an implicit backward-Euler scheme. Several turbulence models are available including the one-equation Spalart-Allmaras (SA) model and several two-equation models. The two-equation models available are the Jones and Launder k- $\varepsilon$ model, Menter Shear Stress Transport (SST) model, nonlinear Algebraic Reynolds Stress Models (ARSM) of Girimaji and Shih/Zhu/Lumley, and Wilcox 1988 k- $\omega$ model. Detached Eddy Simulation (DES) has been implemented in all of the turbulence models. A capability to trip the flow at specified locations on aerodynamic surfaces has been implemented for the k- $\varepsilon$ turbulence model, but fully turbulent flow is assumed for the results in this paper. USM3D has capabilities for overset grids and dynamic grid motion, the latter being utilized in the current study. 
Published guidelines ${ }^{4}$ for computing dynamic forced oscillation solutions on SACCON were employed for the CFD computations in this study. A half-span tetrahedral grid was generated on the SACCON configuration with aftsting using a developmental version of the VGRID $\operatorname{code}^{21}$ with identical spacing parameters as used for Grid 1 in Ref. 4. The half-span grid was mirrored to create a full-span grid with 6.5 million cells. For the dynamic solutions, the entire grid was rotated in solid body motion about the model roll axis at $\alpha=20^{\circ}$ to mimic the wind tunnel procedure.

Selection of amplitude, frequency, and angles of attack were chosen to allow direct comparison with the wind tunnel tests that ranged from $0.24 \mathrm{~Hz}$ to $1.0 \mathrm{~Hz}$. CFD simulation cases were computed at three additional frequencies to expand the range of experimental frequencies, resulting in a total of 10 frequencies, $0.04,0.14,0.24,0.36,0.44$, $0.55,0.66,0.86,1.00$, and $1.20 \mathrm{~Hz}$. The corresponding range for reduced frequencies, $k$, is 0.0106 to 0.3171 . The CFD simulations presented for this study are for small amplitude roll oscillations $\left(\phi_{A}=5^{\circ}\right)$ at one angle of attack $(\alpha$ $=20^{\circ}$ ), where wind tunnel results show strong frequency dependence in Fig. 2. One potential source of uncertainty in CFD calculations is in turbulence modeling. In this study, two turbulence models were used to calculate CFD forced-oscillation simulations: (1) the Spalart-Allmaras (SA) one-equation model and (2) the Menter Shear Stress Transport (SST) two-equation model.

A total of 20 dynamic CFD solutions were generated using the most conservative guidelines from Ref. 4. The cases were computed at $M_{\infty}=0.2$ to improve the solution convergence characteristics. With the assumption of incompressible flow, the actual roll frequencies were adjusted to match the correct reduced frequencies. The dynamics solutions were restarted from two static time-accurate solutions generated with the SA and SST turbulence models at $\alpha=20^{\circ}$. Each case was oscillated about the body axis with $\phi_{A}=5^{\circ}$ at the appropriate reduced frequency through two cycles of sinusoidal motion. The cycles were discretized in time using 1800 steps per cycle and 20 inner iterations (36,000 total iterations per cycle) of the $2^{\text {nd }}$-order physical timestep scheme, which resulted in sample rates between $72 \mathrm{~Hz}$ and $2160 \mathrm{~Hz}$. Because of the underlying Reynolds Averaging assumption for the Navier-Stokes equations, the dynamic solutions are typically converged to a steady hysteresis of rolling moment $C_{l}$ vs. $\phi$ after the first $1 / 4$ to $1 / 2$ cycle. Hence, the last full oscillation cycle is considered converged.

Once the CFD solutions were complete, a special process was applied to transform the CFD data into a format that is compatible with the wind tunnel data for post-processing with the established aerodynamic modeling software. The last full cycle of CFD data at each frequency was replicated 20 times and concatenated to form multiple cycles of data. The corresponding time stamps were incremented for each replicated cycle to create a set of data continuous in time that mimics the wind tunnel data.

\section{Aerodynamic Modeling}

The model equations for the lateral coefficients, $C_{Y}, C_{n}$, and $C_{l}$, represented by $C_{a}$, where $a=Y, n$, or $l$, were developed from a general form of the indicial model equations presented in Refs. 22-23. Each coefficient is considered in the form

$$
C_{a}(t)=C_{a}(0)+\int_{0}^{t} C_{a_{\beta}}(t-\tau) \dot{\beta}(\tau) d \tau+\frac{b}{2 V} \int_{0}^{t} C_{a_{p}}(t-\tau) \dot{p}(\tau) d \tau+\frac{b}{2 V} \int_{0}^{t} C_{a_{r}}(t-\tau) \dot{r}(\tau) d \tau
$$

where $C_{a_{\beta}}(t), C_{a_{p}}(t)$, and $C_{a_{r}}(t)$ are the indicial functions and $C_{a}(0)$ is the initial value of $C_{a}$. Two assumptions were adopted to simplify the model: (a) the effect of angular accelerations $\dot{p}$ and $\dot{r}$ on any coefficient can be neglected and (b) the indicial functions in Eq. (1) can be expressed as

$$
C_{a_{\beta}}(t)=C_{a_{\beta}}(\infty)-F_{a_{\beta}}(t)
$$

where $F_{a_{\beta}}(t)$ is the deficiency function and $C_{a_{\beta}}(\infty)$ is the partial derivative of $C_{a}$ with respect to $\beta$ evaluated in steady flow conditions.

The simplified model, which takes into account changes with respect to steady state, has the form 


$$
C_{a}(t)=C_{a_{\beta}}(\infty) \beta(t)+\frac{b}{2 V} C_{a_{p}}(\infty) p(t)+\frac{b}{2 V} C_{a_{r}}(\infty) r(t)-\int_{0}^{t} F_{a_{\beta}}(t-\tau) \dot{\beta}(\tau) d \tau
$$

where $C_{a}(0)$ has been subtracted from both sides of Eq. (3). To obtain a model appropriate for identification and with a limited number of parameters, the deficiency function is assumed to be a simple exponential function ${ }^{23}$

$$
F_{a \beta}=a e^{-b_{1} t}
$$

Models appropriate for an aircraft undergoing one degree of freedom forced oscillation in roll can be obtained using Eqs. (3) and (4). Considering one degree of freedom rolling motion in the tunnel

$$
C_{a}(t)=C_{a}[\phi(t), p(t)]
$$

where roll angle is related to the sideslip angle by the equation

$$
\beta(t)=\sin ^{-1}(\sin \alpha \sin \phi(t))
$$

Combining Eqs. (3-6), the aerodynamic models can be formulated as

$$
C_{a}(t)=C_{a_{\beta}}(\infty) \beta(t)+\frac{b}{2 V} C_{a_{p}}(\infty) p(t)-a \int_{0}^{t} e^{-b_{1}(t-\tau)} \dot{\beta}(\tau) d \tau
$$

By introducing

$$
\eta(t)=\int_{0}^{t} e^{-b_{1}(t-\tau)} \dot{\beta}(\tau) d \tau
$$

and applying the Leibnitz integral rule, the state space form of Eq. (7) can be written as

$$
\begin{gathered}
\dot{\eta}(t)=-b_{1} \eta(t)+\dot{\beta}(t) \\
C_{a}(t)=C_{a_{\beta}}(\infty) \beta(t)+\frac{b}{2 V} C_{a_{p}}(\infty) p(t)-a \eta(t)
\end{gathered}
$$

From Eq. (7), a steady response can be obtained ${ }^{24}$ as 


$$
C_{a}(t)=\bar{C}_{a \beta} \phi_{A} \sin (\omega t)+\bar{C}_{a_{p}} \phi_{A} k \cos (\omega t)
$$

where $\phi_{A}$ is the amplitude of roll oscillation, $k$ is reduced frequency, and $\bar{C}_{a_{\beta}}$ and $\bar{C}_{a_{p}}$ are the in-phase and out-ofphase components, respectively. These components are related to the model parameters, Refs. 24-25, by the equations

$$
\begin{gathered}
\bar{C}_{a_{\beta}}=C_{a_{\beta}}(\infty) \sin \alpha_{0}-a \frac{\tau_{1}^{2} k^{2}}{1+\tau_{1}^{2} k^{2}} \\
\bar{C}_{a_{p}}=C_{a_{p}}(\infty)-a \frac{\tau_{1}}{1+\tau_{1}^{2} k^{2}} \sin \alpha_{0}
\end{gathered}
$$

\section{Model Identification}

Roll oscillation data from wind tunnel measurements and CFD simulations provide sufficient information for determining adequate models for the lateral aerodynamics. These models have a postulated structure with parameters that can be estimated using harmonic analysis and a Two-Step linear regression method. The last step in model identification is model validation where the models are evaluated as predictors of response data not used previously for identification.

\section{A. Harmonic Analysis}

A method of harmonic analysis, Ref. 26, was applied to measured aerodynamic coefficients. A mathematical model for these coefficients is

$$
C_{a}(t)=A_{0}+\sum_{j=1}^{m} A_{j} \cos (j \omega t)+\sum_{j=1}^{m} B_{j} \sin (j \omega t) \quad a=l, n \text { or } \mathrm{Y}
$$

where $A_{0}, A_{j}$, and $B_{j}$ are the Fourier coefficients. The analysis provides estimates of these coefficients, their standard errors, and the multiple correlation coefficient, $R^{2}$. For the model with linear aerodynamics and $A_{0}=0$, the aerodynamic in-phase and out-of-phase components can be expressed in terms of the coefficients $A_{1}$ and $B_{1}$. For the roll oscillation case the expressions are

$$
\begin{gathered}
\bar{C}_{a_{\beta}}=\frac{B_{1}}{\phi_{A}} \\
\bar{C}_{a_{p}}=\frac{A_{1}}{k \phi_{A}}
\end{gathered}
$$


where $\beta$ is related to $\phi$ by Eq. (6).

The multiple correlation coefficient, $R^{2}$, indicates the fraction of the variation in the measured data explained by the model and is defined as

$$
R^{2}=1-S S_{E} / \mathrm{SS}_{r} \quad 0<R^{2}<1
$$

where

$$
S S_{E}=\sum_{i=1}^{N}\left[C_{a_{E}}(i)-\hat{C}_{a}(i)\right]^{2}
$$

is the residual sum of squares and

$$
S S_{r}=\sum_{i=1}^{N}\left[C_{a_{E}}(i)-\tilde{C}_{a}(i)\right]^{2}
$$

is the total sum of squares. $C_{a_{E}}(i), \hat{C}_{a}(i)$, and $\tilde{C}_{a}(i)$ are the measured, estimated, and mean values, respectively.

Harmonic analysis was performed on roll oscillatory wind tunnel data with amplitude $\phi_{A}=5^{\circ}$. Data were obtained from the study in Ref. 3. Results of the harmonic analysis are presented in Fig. 2. This figure shows both out-of-phase and in-phase components of the roll moment and the multiple correlation coefficient against angle of attack at different frequencies. The results show little or no frequency dependence for angles of attack at $15^{\circ}$ and below and therefore no unsteady aerodynamic effect. At $20^{\circ}$ angle of attack frequency dependence is clearly indicated.

Figure 2 also reveals some variation and lower values of $R^{2}$ for angle of attacks at 14 and $15^{\circ}$. Lower values of $R^{2}$ indicate reduced adequacy of the linear model representation given by conventional in-phase and out-of-phase coefficients; however, practical experience suggests that values of $R^{2} \geq 0.8$ are sufficient to accept the linear model structure. As follows from Eqs. (17-19), the estimates of $R^{2}$ are influenced by the value and number of Fourier coefficients (harmonic order) in Eq. (14) as well as the measurement noise in $C_{a_{E}}(i)$. Because the Fourier coefficients are mutually orthogonal, the estimates of $A_{j}$ and $B_{j}$ will not change with the number of coefficients included in Eq. (14). Changes will only appear in the corresponding standard errors and residuals defined by Eq. (18). This makes $R^{2}$ an effective diagnostic tool to discern the adequacy of a linear first-order model against nonlinear higher harmonic models. Higher order models to capture aerodynamic nonlinearities are not necessary in this case.

Figure 3 shows the behavior of out-of-phase and in-phase components as a function of frequency at $\alpha_{0}=20^{\circ}$. Since the design of this wind tunnel investigation was not intended to investigate unsteady behaviors specifically, the range of lower frequencies is limited. In general, this can limit the information content for general modeling and identification. Over a wide range of frequencies these terms will typically show an asymptotic behavior reaching a constant value for high frequencies and larger values for lower frequencies. Some of this character is visible in the figure for the wind tunnel data but additional frequencies would demonstrate this effect more clearly.

Fortunately additional data extending the frequency range and demonstrating this expected relationship was obtained using CFD simulations. Figure 3 also shows the CFD results for both the SA and SST turbulence models. These results show increasing values for lower frequencies and relatively constant or asymptotic behavior for higher frequencies. These results represent an intermediary step in the modeling process that can be directly compared with wind tunnel results. Future wind tunnel tests will be required to obtain comparable lower frequency data. 


\section{B. Parameter Estimation Using Two-Step Linear Regression}

Parameter estimation was accomplished using the Two-Step Linear Regression (LR) method. This regression method is applied in the frequency domain using Eqs. (12) and (13). Specific application of this method is described in Refs. 18, 26-27. The LR method is used for the general case where both in-phase and out-of-phase components present frequency dependence and a linear aerodynamic model is adequate. General application of regression and other techniques for aircraft are explained and relevant software is provided in Ref. 28.

An aerodynamic model of an aircraft performing a one degree-of-freedom oscillatory motion about one of its body axes can be formulated in terms of the in-phase and out-of-phase components ${ }^{14}$ for rolling motion as

$$
\begin{gathered}
\bar{C}_{a_{\beta}}=C_{a_{\beta}}(\infty) \sin \alpha-a f_{1} \sin \alpha \\
\bar{C}_{a_{p}}=C_{a_{p}}(\infty)-a f_{0} \sin \alpha
\end{gathered}
$$

where

$$
\begin{aligned}
& f_{1}=\frac{\tau_{1}^{2} k^{2}}{1+\tau^{2} k^{2}} \\
& f_{0}=\frac{\tau_{1}}{1+\tau_{1}^{2} k^{2}}
\end{aligned}
$$

and subscript $a$ denotes appropriate force or moment coefficient. With the expression,

$$
\frac{\tau_{1}^{2} k^{2}}{1+\tau_{1}^{2} k^{2}}=1-\frac{1}{1+\tau_{1}^{2} k^{2}}
$$

Eq. (20) can be rearranged into a set of equations for $\mathrm{m}$ different values of $\mathrm{k}$ as

$$
y(j)=a_{0}+a_{1} x(j), \quad j=1,2, \ldots, m
$$

where for rolling oscillations

$$
\begin{aligned}
& x=\bar{C}_{a_{\beta}}, y=\bar{C}_{a_{p}} \\
& a_{0}=C_{a_{p}}(\infty)+a_{1}\left(a+C_{a_{\beta}}\right) \sin \alpha \\
& a_{1}=-\tau_{1}
\end{aligned}
$$

In the first step a linear regression is used in estimation of parameters $a_{0}$ and $a_{1}$ in Eq. (23) from measured in-phase and out-of-phase components at $m$ different frequencies, $m>2$.

The second step of regression follows from Eqs. (20-21) replacing $\tau_{1}$ by its estimated value. The resulting equations are 


$$
\begin{aligned}
& y_{1}(j)=d_{0}+d_{1} x_{1}(j) \\
& y_{2}(j)=c_{0}+d_{1} x_{2}(j)
\end{aligned}
$$

where for rolling oscillations these terms are

$$
\begin{gathered}
y_{1}(j)=\bar{C}_{a_{\beta}}(j), \quad y_{2}(j)=\bar{C}_{a_{p}}(j) \\
x_{1}(j)=-f_{1}(j) \sin \alpha, \quad x_{2}(j)=-f_{0}(j) \sin \alpha \\
d_{0}=C_{a_{\beta}}(\infty) \sin \alpha, \quad d_{1}=a, \quad c_{0}=C_{a_{p}}(\infty)
\end{gathered}
$$

Similar expressions are obtained for pitching and yawing oscillations. More discussion on the development of regression equations and estimator properties can be found in Ref. 28.

Table I. LR parameter estimates, from wind tunnel, for unsteady model at $\alpha_{0}=20^{\circ}$ and $\alpha_{A}=5^{\circ}$.

\begin{tabular}{|c|c|c|c|c|c|}
\hline & \multicolumn{2}{|c|}{ Step 1 } & \multicolumn{3}{c|}{ Step 2 } \\
\hline & $b_{1}$ & $\tau_{1}$ & $a$ & $C_{l p}$ & $C_{l \beta}$ \\
\hline$\hat{\theta}$ & 3.68 & 6.37 & 0.75 & -0.40 & -0.57 \\
\hline$\hat{\sigma}(\theta)$ & $(0.34)$ & $(0.58)$ & $(0.04)$ & $(0.47)$ & $(0.05)$ \\
\hline
\end{tabular}

The LR method was applied to roll oscillatory data from NASA Langley $14 \times 22$ Wind Tunnel experiment using the SACCON aircraft. Parameter estimates and their standard errors are provided in Table I. To check model fit, estimated in-phase and out-of-phase components can be calculated by applying parameter estimates from Table I to Eqs. (11-13) and comparing with the wind tunnel values. One approach for checking model fit and consistency of the linear relationship in Eq. (23) at the same time is to plot the out-of-phase component against the in-phase component for both the measured values and estimated values. The slope of these data is equal to the time constant $\tau_{1}$. Figure 4 shows the model fit and consistency check based on wind tunnel data. The figure shows a very good fit of the model to the data as well as a satisfactory linear relationship between in-phase and out-of-phase components.

Applying the same estimation process to CFD simulated data provides similar model checks. Figs. 5-6 show these results for the SA and SST turbulence models, respectively. In this case the SA model appears to satisfy the linear relationship except for the lowest frequency data point. The low frequency data point may be an outlier or it may reflect an aerodynamic characteristic not captured by the current model structure. Investigating this particular data point is beyond the scope of the current study; further wind tunnel tests are required to check the validity of this point. For purposes of comparing models in this study and respecting the benefits of a parsimonious regression model, it will be retained. The impact will be larger uncertainty bounds on the estimated parameters. Results for the SST case show the same characteristic for the low frequency data point and, in addition, a more quadratic shape with frequency. For purposes of this study the linear model structure is accepted along with increased model uncertainty.

A direct comparison of the four model parameters and associated $2 \sigma$ standard deviations is shown in Fig. 7 for wind tunnel, SA, and SST cases. For the wind tunnel case, uncertainty in the model parameters is derived from measurement noise and model error. For the CFD cases, uncertainty is derived only from model error; however two sources of model error are presented. The first source of model error is due to differences in the linear unsteady model representation of SA or SST data. This error is reflected in the $2 \sigma$ bounds for each case. The second source of model error results from the choice of the two turbulence models. The two turbulence models represent a range of model choice that is part of the model uncertainty. The charts for $C_{l_{p}}$ and $C_{l_{\beta}}$, in Fig. 7, show the uncertainty bars overlapping for all three cases. The implication is that no statistical difference exists between cases for these parameters, at the $95 \%$ confidence level. This result is for a single-case study; consequently the results are not under statistical process control as discussed in Ref. 2. Many more replications of the three cases would be needed for that 
purpose. However, when that option is not available, it is useful to account for any uncertainty within a single case study as demonstrated in Fig. 7. The two charts on the right side of Fig. 7 show the parameters for the unsteady component of the model. The time constant $\tau_{l}$ for the wind tunnel and SA cases are in statistical agreement but the SST case produced a statistically different result. For the attenuation factor, $a$, the SA and SST are in statistical agreement but not with the wind tunnel case.

\section{Model Validation}

Validation of models from wind tunnel tests are demonstrated through prediction of dynamic responses using measured data not used for model identification. Ideally, a substantially different input would be used during the experiment to create validation measurements. For example, a step input provides a suitable test for the new unsteady models that have been estimated from sinusoidal data. For these wind tunnel tests step inputs were not an option since the LaRC $14 \times 22$ dynamic test rig can only produce sinusoidal motion. One option in this case is to create a validation data set by removing one frequency case from the estimation process and using it as a validation test case. For this case, no significant affect on the estimated model parameters occurs by removing one frequency. To demonstrate the model prediction capability of the wind tunnel derived model, a comparison of measured and predicted rolling moment coefficient is shown in Fig. 8. The highest and lowest frequencies for the low, middle, and high angles of attack are shown. These cases show the largest range of responses obtained from the wind tunnel tests. All the model fits to the measured data are very good indicating an adequate model. For at $\alpha_{0}=20^{\circ}$, response prediction is based on the LR unsteady model and for $\alpha_{0}<20^{\circ}$ the responses are based on the harmonic models.

Validation of the SA and SST LR models for the unsteady case at $\alpha_{0}=20^{\circ}$, are demonstrated by a comparison of measured and predicted rolling moment coefficient as shown in Fig. 9. Time histories at the highest and lowest frequencies are compared with the wind-tunnel LR model prediction and measured rolling moment data. The SA model is in very good agreement with the wind tunnel model and provides a good fit to the measured data. The SST model shows some difference with the both SA and wind tunnel measurements. In spite of steady damping terms from wind tunnel and SST models being in reasonable agreement the differences in the unsteady components are sufficient to show response prediction differences. Differences between SA and SST model parameters and response predictions reflect the range of uncertainty present due to selection of turbulence models.

\section{Concluding Remarks}

This paper presented two methods, harmonic analysis and two a two-step linear regression, for estimating mathematical models useful for stability and control analysis that can be applied to both CFD simulations and wind tunnel measurements. This approach offers a model structure that can accommodate unsteady aerodynamic behavior and characterize model parameter uncertainty. Conventional stability and control derivatives are retained in the recommended models to take advantage of the knowledge base built up through years of aerospace engineering practice. Application of harmonic analysis demonstrated that unsteady behavior was present in the rolling moment at $\alpha=20^{\circ}$ and both wind tunnel measurements and CFD simulated data confirmed this result. Linear regression produced linear unsteady models at the same angle of attack. Model parameters between the wind tunnel and CFDSA models were in statistical agreement at the $95 \%$ confidence level, except for the " $a$ " parameter which is a gain or multiplicative factor on the unsteady term. Regression analysis of the CFD-SST simulated data resulted in a steadyflow damping parameter in statistical agreement with the wind tunnel and CFD-SA cases but not for the unsteady term. For the CFD-SST case, the unsteady term was statistically different and with a much larger uncertainty bound that either wind tunnel or CFD-SA cases. Time history comparisons confirm the model agreement between the wind tunnel and CFD-SA models but the CFD-SST case shows the added uncertainty due to turbulence model selection. These results highlight the importance of experiment design considerations to expand wind tunnel test inputs that cover an appropriately wide range of frequencies and to provide additional inputs different from sinusoidal motion.

This paper suggests an approach for model validation using system identification to identify models from CFD simulations and wind tunnel measurements. By presenting a modeling methodology that determines an adequate model from both sources of data, the approach creates a focus on key aerodynamic model parameters and their uncertainties as a method for comparison and extends conventional validation methods that rely solely on time history comparisons.

\section{Acknowledgments}

The authors extend their appreciation to the NASA Aviation Safety Program, Vehicle Systems Safety project, and the NASA Fundamental Aerodynamics Program, Subsonic Fixed Wing project, for the opportunity to support the NATO RTO AVT-161 task group. 


\section{References}

${ }^{1}$ Morrison, Joseph H., and Hemsch, Michael J., "Statistical Analysis of CFD Solutions from the Third AIAA Drag Prediction Workshop," AIAA 2007-254, January 2007.

${ }^{2}$ Hemsch, Michael J., "Statistical Analysis of Computational Fluid Dynamics Solutions from the Drag Prediction Workshop," Journal of Aircraft, Vol. 41, No. 1, January-February, 2004.

${ }^{3}$ Vicroy, D. and Loeser, T., "SACCON Dynamic Wind Tunnel Tests at DNW- NWB and 14'x22' NASA LaRC", AIAA2010-4394, June 2010.

${ }^{4}$ Frink, N.T., "Strategy for Dynamic CFD Simulations on SACCON Configuration”, AIAA 2010-4559, June 2010.

${ }^{5}$ Cummings, R. and A. Schütte, A., “An Integrated Computational/Experimental Approach to UCAV Stability \& Control Estimation: Overview of NATO RTO AVT- 161”, AIAA-2010-4392, June 2010.

${ }^{6}$ Loeser, T., Vicroy, D., and Schütte, A., "SACCON Static Wind Tunnel Tests at DNW- NWB and 14'x22' NASA LaRC", AIAA-2010-4393, June 2010.

${ }^{7}$ Gilliot, A., "Static and Dynamic SACCON PIV Tests - Part I: Forward Flowfield", AIAA-2010-4395, June 2010.

${ }^{8}$ Konrath, R., Roosenboom, E., Schröder, A., Pallek, D., and Otter, D., "Static and Dynamic SACCON PIV Tests - Part II: Aft Flow Field", AIAA-2010-4396, June 2010.

${ }^{9}$ Vallespin, D., Boelens, O. and Badcock, K., "SACCON CFD Simulations Using Structured Grid Approaches", AIAA2010-4560, June 2010.

${ }^{10}$ Tormalm, M. Tomac, M. and Schmidt, S., "Computational Study of Static And Dynamic Vortical Flow over the Delta Wing SACCON Configuration Using the FOI Flow Solver Edge”, AIAA-2010-4561, June 2010.

${ }^{11}$ Le Roy, J. "SACCON CFD Static and Dynamic Derivatives Using elsA", AIAA-2010-4562, June 2010.

${ }^{12}$ Chakravarthy S. and Chi, D., "SACCON CFD Simulations Using CFD++", AIAA-2010-4563, June 2010.

${ }^{13}$ Schütte, A., Hummel, D. and Hitzel, S. "Numerical and Experimental Analyses of the Vortical Flow Around the SACCON Configuration", AIAA-2010-4690, June 2010.

${ }^{14}$ Cummings, R. Petterson, K. Jirasek, A. and Schmidt, S., "SACCON Static and Dynamic Motion Flow Physics Simulations Using COBALT", AIAA-2010-4691, June 2010.

${ }^{15}$ Rohlf, D., Schmidt, S., and Irving, J., "SACCON Stability and Control Analysis Applying System Identification Techniques", AIAA 2010-4399, June 2010.

${ }^{16}$ Murphy, P.C. and Klein, V., "Estimation of Aircraft Unsteady Aerodynamic Parameters from Dynamic Wind Tunnel Testing," AIAA 2001-4016, August 2001.

${ }^{17}$ Murphy, P.C. and Klein, V., "Validation of Methodology for Estimating Aircraft Unsteady Aerodynamic Parameters from Dynamic Wind Tunnel Tests," AIAA 2003-5397, August 2003.

${ }^{18}$ Murphy, P.C. and Klein, V., "Transport Aircraft System Identification Using Roll and Yaw Oscillatory Wind Tunnel Data", AIAA 2010-8122, August 2010.

${ }^{19}$ Frink, N. T., "Tetrahedral Unstructured Navier-Stokes Method for Turbulent Flows," AIAA Journal, Vol. 36, No. 11, November 1998, pp. 1975-1982.

${ }^{20}$ Frink, N. T., Pirzadeh, S. Z., Parikh , P. C., Pandya, M. J.(2000) “The NASA Tetrahedral Unstructured Software System”. Aeronautical Journal, Vol. 104, No. 1040 (491-499). TetrUSS website: http://tetruss.larc.nasa.gov.

${ }^{21}$ Pirzadeh, S. Z., "Advanced unstructured grid generation for complex aerodynamic applications," AIAA Journal, Vol. 48, No. 5, 2010, pp. 904-915.

${ }^{22}$ Jones, Robert T. and Fehlner, Leo F., "Transient Effects of the Wing Wake on the Horizontal Tail," NACA TN-771, 1940.

${ }^{23}$ Tobak, Murray, "On the Use of Indicial Function Concept in the Analysis of Unsteady Motions of Wings and Wing-Tail Combinations, NACA Rep. 1188, 1954.

${ }^{24}$ Klein, Vladislav and Norderer, Keith D., "Modeling of Aircraft Unsteady Aerodynamic Characteristics. Part I - Postulated Models," NASA TM 109120, May, 1994.

${ }^{25}$ Klein, Vladislav, "Modeling of Longitudinal Unsteady Aerodynamics of a Wing-Tail Combination," NASA CR-1999209547, September, 1999.

${ }^{26}$ Klein, Vladislav, Murphy, P.C., and Szyba, Nathan M., "Analysis of Wind Tunnel Lateral Oscillatory Data of the F-16XL Aircraft," NASA/TM-2004-213246, 2004.

${ }^{27}$ Murphy, Patrick C., Klein, Vladislav, and Szyba, Nathan M., "Progressive Aerodynamic Model Identification From Dynamic Water Tunnel Test of the F-16XL Aircraft," AIAA Atmospheric Flight Mechanics Conference, AIAA 2004-5277, AIAA Washington, DC, 2004.

${ }^{28}$ Klein, Vladislav and Morelli, Eugene, "Aircraft System Identification: Theory and Practice," 1st edition, AIAA Inc., Reston, Virginia, 2006. 


\section{Figures}

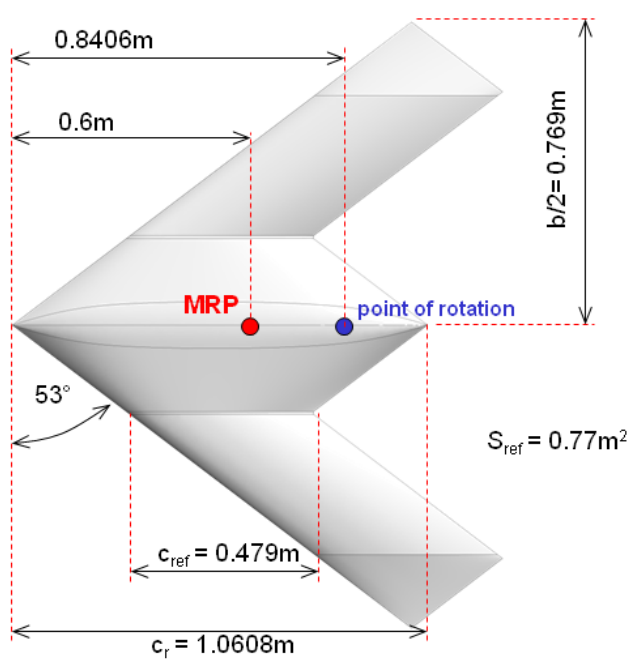

$\mathrm{S}=0.77 \mathrm{~m}^{2}, \bar{c}=0.479 \mathrm{~m}, \mathrm{~b} / 2=0.769 \mathrm{~m}$

Figure 1. Model Geometry for $\mathrm{SACCON}^{3}$ sub-scale aircraft.

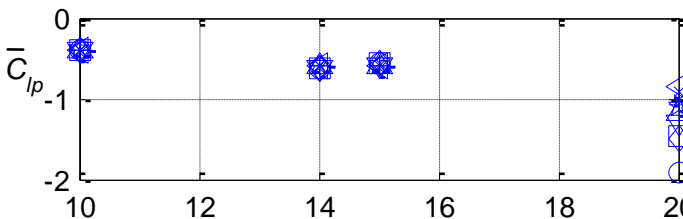

$f(\mathrm{~Hz})$

0.24

$\square \quad 0.36$

$\diamond \quad 0.44$
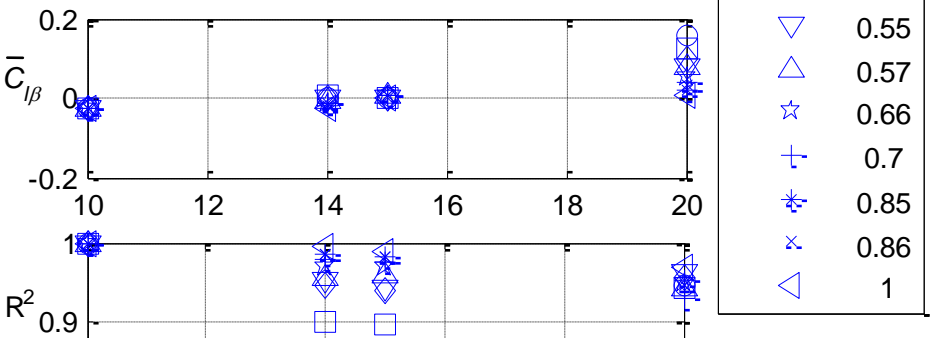

0.8

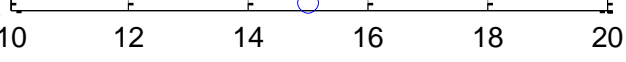

$\alpha, \operatorname{deg}$

Figure 2. Roll moment out-of-phase and in-phase components from harmonic analysis of wind tunnel forced oscillations in roll, $\phi_{A}=5^{\circ} . \mathrm{SACCON}^{3}$.
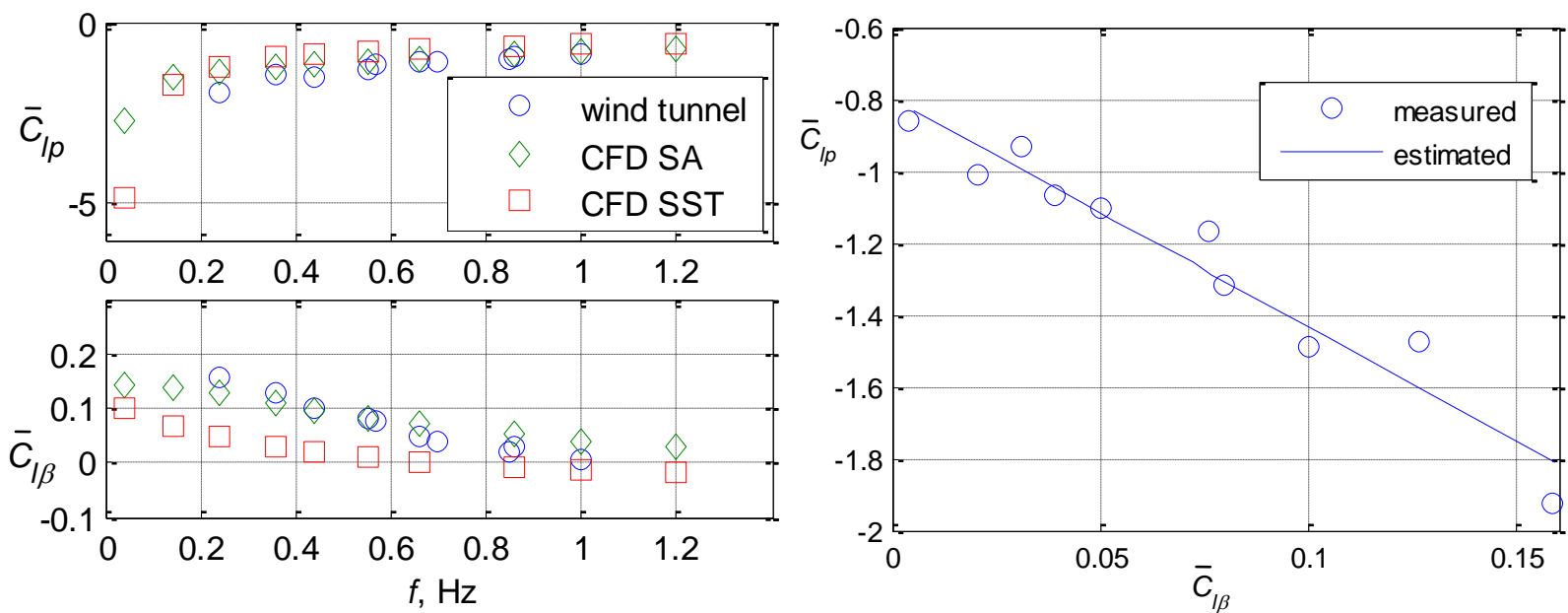

Figure 3. Variation of out-of-phase and in-phase components with frequency at $20^{\circ}$ angle of attack. Roll oscillatory data, $\phi_{A}=5^{\circ}$. $\mathrm{SACCON}^{3}$.

Figure 4. LR model estimates from roll oscillatory wind tunnel data at $20^{\circ}$ angle of attack, $\phi_{A}=5^{\circ}$. SACCON ${ }^{3}$.

12

American Institute of Aeronautics and Astronautics 


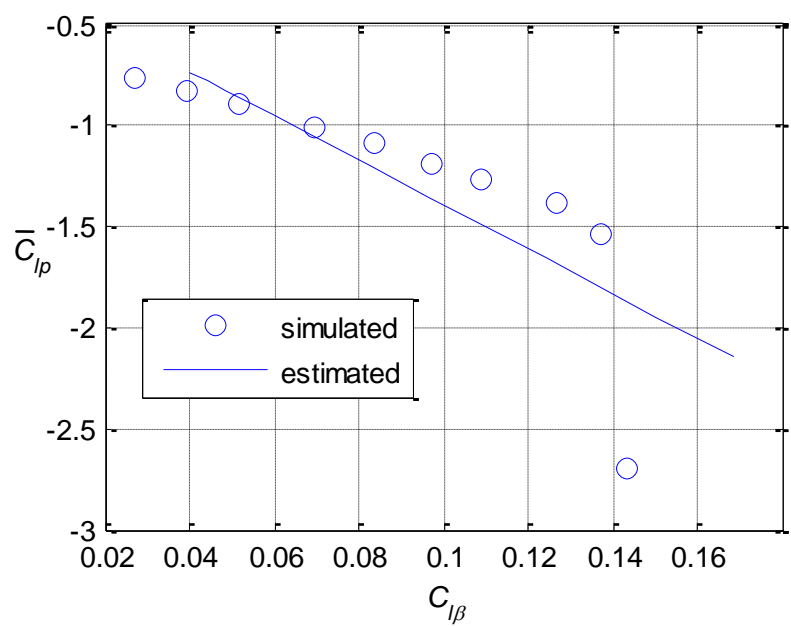

Figure 5. Out-of-phase vs in-phase component at $20^{\circ}$ angle of attack. CFD roll oscillatory data using SA turbulence model, $\phi_{A}=5^{\circ}$. SACCON ${ }^{3}$.

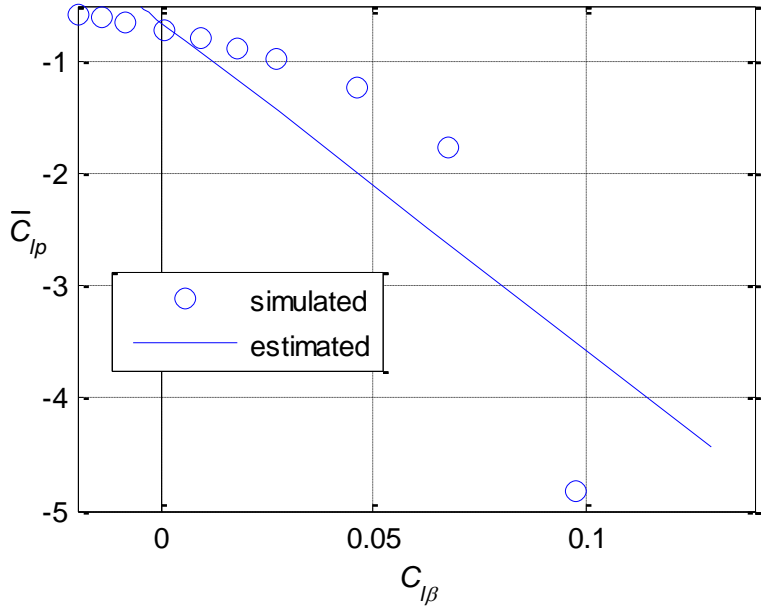

Figure 6. Out-of-phase vs in-phase component at $20^{\circ}$ angle of attack. CFD roll oscillatory data using SST turbulence model, $\phi_{A}=5^{\circ}$. SACCON ${ }^{3}$.
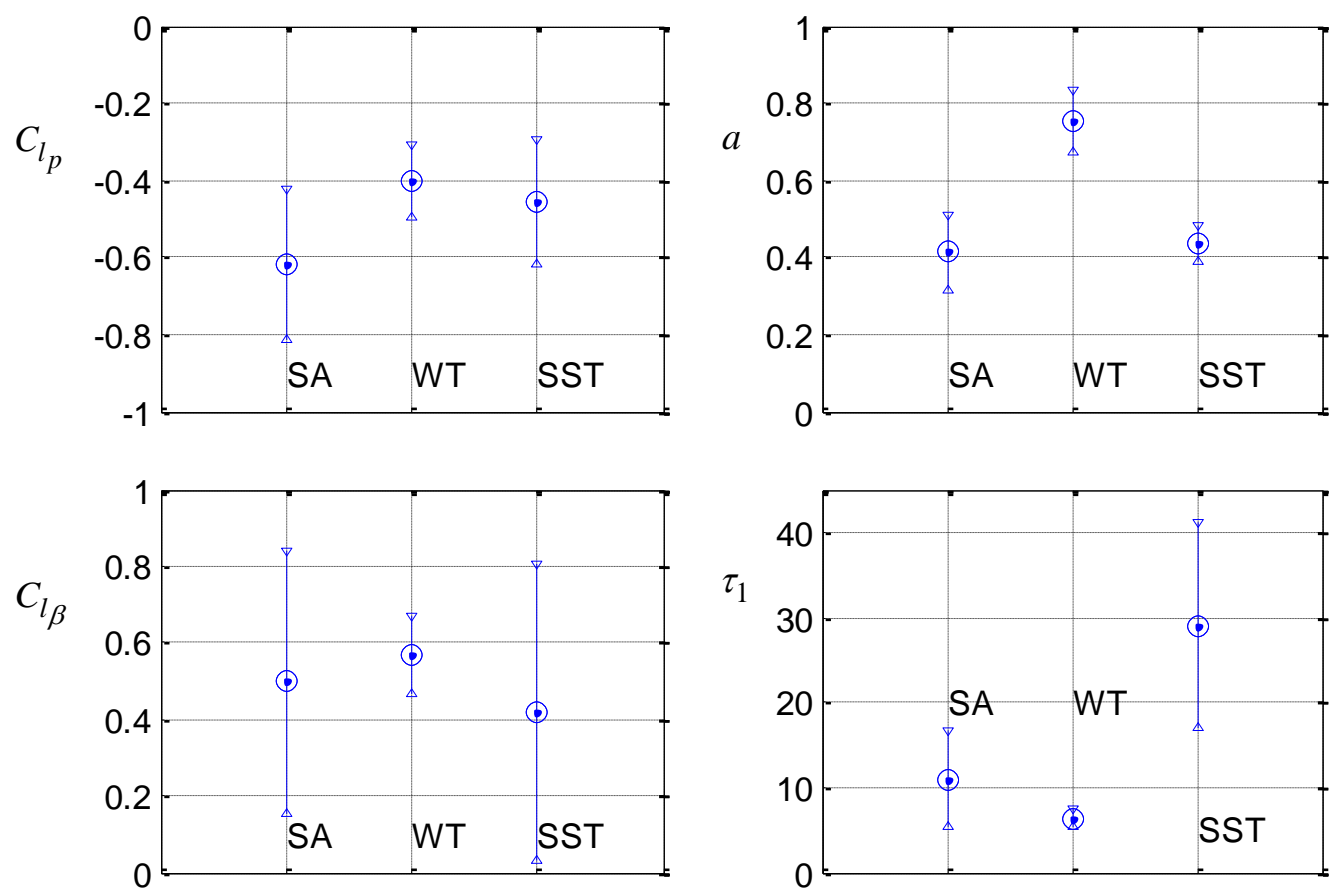

Figure 7. Two-Step model parameter estimates and $2 \sigma$ error bounds, at $\alpha=\mathbf{2 0}$, using wind tunnel and CFD roll oscillatory data, $\phi_{A}=5^{\circ}, \mathrm{SACCON}^{3}$. 

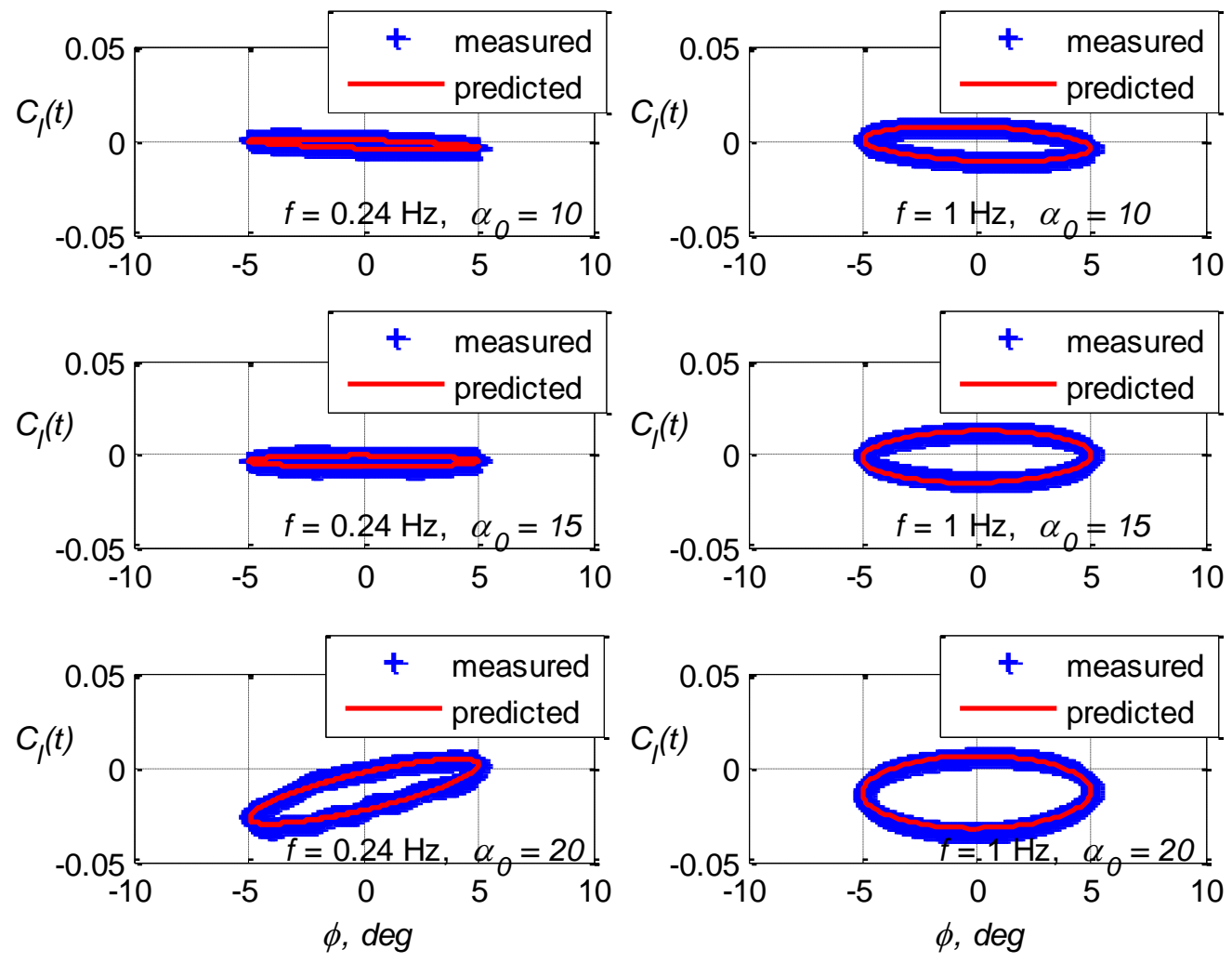

Figure 8. Harmonic model prediction $(\mathbf{a}<20)$ and Two-Step Model prediction $(\mathbf{a}=20)$. Models from wind tunnel roll oscillatory data, $\phi_{A}=5^{\circ}, \mathrm{SACCON}^{3}$.
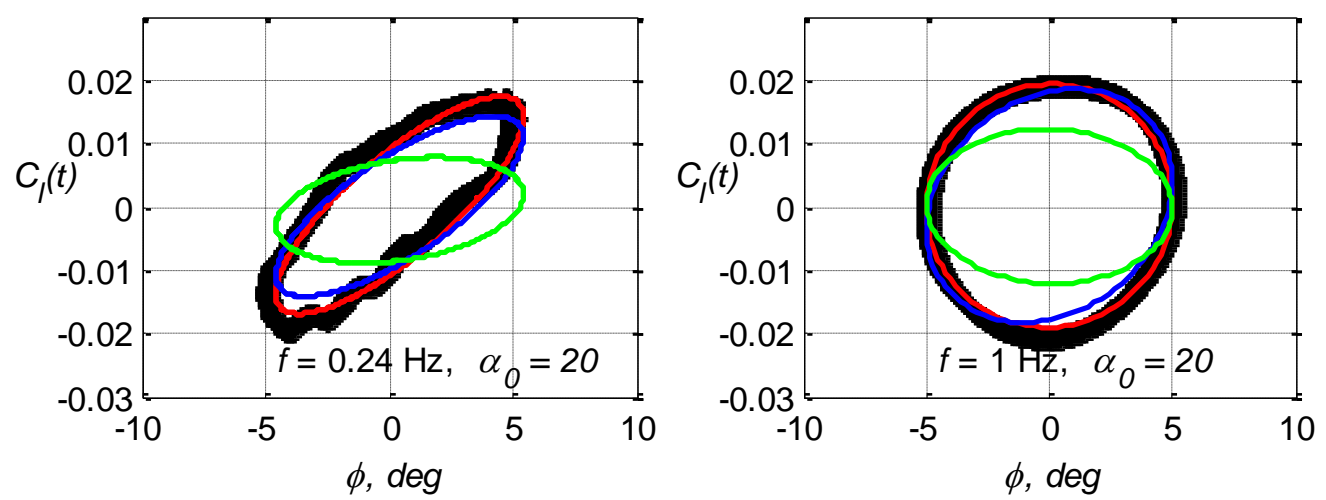

Figure 9. Model response predictions at $\alpha=20^{\circ}$ for roll oscillatory data, $\phi_{A}=5^{\circ}$, SACCON ${ }^{3}$. Key: Black $=$ WT measurements, Red $=$ Two-Step model from WT, Blue $=$ Two-Step model from CFD-SA, Green $=$ Two-Step model from CFD-SST . 